\title{
Annex IX
}

\section{Small Ape Population Decline by Taxon, in Descending Order of Abundance}

\begin{tabular}{|c|c|c|c|}
\hline Taxon & Abundance & Annual rate of change & Total estimated change, 1973-2018 \\
\hline $\begin{array}{l}\text { Bornean white-bearded gibbon } \\
\text { Hylobates albibarbis }\end{array}$ & 120,000 & -1.54 & $-50 \%$ \\
\hline $\begin{array}{l}\text { Bornean gray gibbon } \\
\text { Hylobates funereus }\end{array}$ & 100,000 & -1.54 & $-50 \%$ \\
\hline $\begin{array}{l}\text { Müller's gibbon } \\
\text { Hylobates muelleri }\end{array}$ & 100,000 & -1.54 & $-50 \%$ \\
\hline $\begin{array}{l}\text { Pileated gibbon } \\
\text { Hylobates pileatus }\end{array}$ & 60,000 & $>-1.54$ & $>-50 \%$ \\
\hline $\begin{array}{l}\text { Siamang } \\
\text { Symphalangus syndactylus }\end{array}$ & 60,000 & -1.73 & $-50 \%$ \\
\hline $\begin{array}{l}\text { Moloch gibbon } \\
\text { Hylobates moloch }\end{array}$ & 48,500 & -1.54 & $-50 \%$ \\
\hline $\begin{array}{l}\text { Gaoligong hoolock } \\
\text { Hoolock tianxing }\end{array}$ & 40,000 & -3.57 & $-80 \%$ \\
\hline $\begin{array}{l}\text { Agile gibbon } \\
\text { Hylobates agilis }\end{array}$ & 25,000 & $>-1.54$ & $>-50 \%$ \\
\hline $\begin{array}{l}\text { Kloss's gibbon } \\
\text { Hylobates klossii }\end{array}$ & 25,000 & -1.54 & $-50 \%$ \\
\hline $\begin{array}{l}\text { Lar gibbon } \\
\text { Hylobates lar }\end{array}$ & 25,000 & -1.54 & $-50 \%$ \\
\hline $\begin{array}{l}\text { Western hoolock } \\
\text { Hoolock hoolock }\end{array}$ & 15,000 & -1.54 & $-50 \%$ \\
\hline $\begin{array}{l}\text { Eastern hoolock } \\
\text { Hoolock leuconedys }\end{array}$ & 10,000 & -0.79 & $-30 \%$ \\
\hline $\begin{array}{l}\text { Southern yellow-cheeked crested gibbon } \\
\text { Nomascus gabriellae }\end{array}$ & 8,000 & -1.54 & $-50 \%$ \\
\hline $\begin{array}{l}\text { Northern yellow-cheeked crested gibbon } \\
\text { Nomascus annamensis }\end{array}$ & 6,500 & -1.54 & $-50 \%$ \\
\hline $\begin{array}{l}\text { Southern white-cheeked crested gibbon } \\
\text { Nomascus siki }\end{array}$ & 6,000 & -3.57 & $-80 \%$ \\
\hline $\begin{array}{l}\text { Western black crested gibbon } \\
\text { Nomascus concolor }\end{array}$ & 5,350 & -3.57 & $-80 \%$ \\
\hline $\begin{array}{l}\text { Northern white-cheeked crested gibbon } \\
\text { Nomascus leucogenys }\end{array}$ & 2,000 & -3.57 & $-80 \%$ \\
\hline $\begin{array}{l}\text { Cao Vit gibbon } \\
\text { Nomascus nasutus }\end{array}$ & 229 & -3.57 & $-80 \%$ \\
\hline
\end{tabular}


Hainan gibbon

Nomascus hainanus

Abbott's gray gibbon

Hylobates abbottii
34

$-3.57$

$-1.54$
$-80 \%$

$-50 \%$

Note: A number of taxa experienced similar levels of decline over the 45 year survey period, resulting in the same annual rate of change.

Sources: unpublished IUCN Red List updates, seen by the authors, 2019 (now published in: Brockelman and Geissmann, 2019, 2020; Brockelman et al., 2020; Brockelman, Molur and Geissmann, 2019; Cheyne and Nijman, 2020; Fan, Turvey and Bryant, 2020; Geissmann and Bleisch, 2020; Geissmann et al., 2020; Liswanto et al., 2020; Marshall, Nijman and Cheyne, 2020a, 2020b; Nguyen et al., 2020; Nijman, 2020; Nijman, Cheyne and Traeholt, 2020; Nijman et al., 2020; Pengfei et al., 2020; Rawson et al., 2020a, 2020b, 2020c; Thinh et al., 2020) 